\title{
Validité de structure des échelles psychométriques en santé bucco-dentaire : exemple de la perception de la santé bucco-dentaire en population générale
}

\section{Structure validity of psychometric scales in oral health: example of the perception of oral health in general population}

\author{
Mohamad Hamad ${ }^{1}$, Davit Varron ${ }^{2}$, Frederic Denis ${ }^{3}$, Mounir Mesbah ${ }^{4}$, Stéphanie Tubert- \\ Jeannin $^{5}$, Nathalie Rude ${ }^{6}$
}

\footnotetext{
${ }^{1}$ Laboratoire de Recherches Intégratives en Neurosciences et Psychologie Cognitive, Université Bourgogne FrancheComté, F-25000, Besançon, France, mohammad.hamad@hotmail.com

${ }^{2}$ Laboratoire de Mathématiques de Besançon, UMR CNRS 6623, Université de Bourgogne Franche-Comté, 25000 Besançon, France, davit.varron@univ-fcomte.fr

${ }^{3}$ EA 75-05 Education, Ethique, Santé, Faculté de Médecine, Université François-Rabelais, 37000 Tours, France, frederic.denis@univ-tours.fr

${ }^{4}$ Laboratoire de Probabilités, Statistique et Modélisation (LPSM, UMR8001), Université de Sorbonne, Paris VI, Paris 75006, France, mounir.mesbah@sorbonne-universite.fr

${ }^{5}$ University Clermont Auvergne, EA4847 CROC Centre for Clinical Research in Dentistry, BP10448, 63000 ClermontFerrand, France, stephanie.tubert@uca.fr

${ }^{6}$ Laboratoire de Recherches Intégratives en Neurosciences et Psychologie Cognitive, Université Bourgogne FrancheComté, F-25000, Besançon, France, nathalie.retel-rude@univ-fcomte.fr
}

RÉSUMÉ. Dans cet article, on montre que la validité de structure est une étape essentielle dans la validation psychométrique des échelles de mesure subjectives, le modèle de crédit partial est utilisé pour étudier la validité de structure. On prend comme exemple, la validation psychométrique de l'échelle GOHAI (General Oral Health Assessment Index) pour la population française générale. Le modèle de crédit partial vérifie l'unidimensionnalité de l'échelle GOHAI.

ABSTRACT. In this article, we show that structure validity is an essential step in the psychometric validation of subjective measurement scales, the partial credit model is used to study the structure validity. We take as an example, the psychometric validation of the GOHAI scale (General Oral Health Assessment Index) for the general French population. The partial credit model verifies the unidimensionality of the GOHAI scale.

MOTS-CLÉS. Validation psychométrique, Validité de structure, Modèle de crédit partiel, Unidimensionnalité, GOHAI.

KEYWORDS. Psychometric validation, Structure validity, Partial credit model, Unidimensionality, GOHAI.

\section{Introduction}

Les mesures subjectives, telles que la qualité de vie et les échelles d'évaluation de la satisfaction en matière de soins de santé, utilisent des méthodes de validation psychométrique avant de pouvoir être utilisées. Ces mesures visent à déterminer les caractéristiques des concepts étudiés, afin de préciser les programmes d'amélioration de la santé à mettre en œuvre et à évaluer.

Dans le cadre de l'analyse psychométrique, des variables non mesurables sont utilisées. La difficulté principale porte sur la construction d'un outil permettant de mesurer cette variable - ou trait latent c'est-à-dire le concept à mesurer. Afin de développer un outil adapté, la génération et la sélection d'items sont des étapes fondamentales pour créer un questionnaire hypothétique [Nun78]. En créant l'échelle de mesure subjective, la recherche qualitative est une étape essentielle qui précède la phase de validation psychométrique [Pop00].

Ainsi, les propriétés du caractère latent à évaluer, en fonction de la qualité et de l'exhaustivité des items, constituent la validation du contenu de l'échelle. Par conséquent, une validation psychométrique 
complète évalue la reproductibilité, la reliabilité et la sensibilité d'un questionnaire. Ces trois qualités n'ont pas été traitées dans cet article, qui met l'accent sur la validité de structure.

Dans la littérature, plusieurs méthodes de validation psychométrique existent. Il existe de nombreuses études basées sur la cohérence interne (Cronbach alpha) comme statistique de validation des échelles. Par exemple, la validation psychométrique de l'échelle SCQH [Bla19], la validation psychométrique de l'échelle GOHAI [Tub03] et la validation psychométrique de l'échelle 4-HCS [Bel20]... D'autres études de validation psychométrique sont basées sur des analyses factorielles, par exemple, "the Second Victim Experience and Support Tool" (C-SVEST)[Che19], "the Brief Pain Inventory for chronic nonmalignant pain" [Tan04] et "questionnaire to evaluate nurses'adherence to recommendations for preventing pressure ulcers" (QARPPU)[Moy17]...

Par conséquent, il reste nécessaire de déterminer une procédure commune pour la validation des échelles de mesure subjectives et de concentrer sur la validité de structure de l'échelle.

Parmi les mesures de la santé perçue, les mesures de la santé bucco-dentaire reflètent la perception des patients de leur état bucco-dentaire et devraient inclure des facteurs sociaux et émotionnels (Allen 2003). L'indice d'évaluation de la santé bucco-dentaire gériatrique (GOHAI) est utilisé en santé buccodentaire [Atc90], l'échelle GOHAI comprend douze items (question), ces items évaluent les fonctions physiques, les aspects psychosociaux et les symptômes liés à la présence de maladies dentaires [Den17]. Il existe cinq modalités de réponse pour chaque item et un score a été attribué à chaque modalité de réponse. Le score GOHAI est la somme des réponses aux 12 items.

Dans cet article, on prend comme exemple, la validation psychométrique de l'échelle GOHAI à partir de l'observation de 260 patients de la population générale française, population initialement observée pour la validation de l'échelle GOHAI en France par Tubert-Jeannin et al. [Tub03]. Plus précisément, on étudie la structure de l'échelle GOHAI et souhaitons confirmer si on mesure les concepts qu'on souhaite mesurer à l'aide de nos items, ou si on mesure d'autres sous-concepts.

La validation psychométrique des échelles de qualité de vie est apparue au XXe siècle et a évolué rapidement au cours de ce siècle. L'histoire de la validation psychométrique a commencé avec Spearman (1907) [Spe07], qui a travaillé sur des données qualitatives en classant chaque modalité de réponse. Plus tard, Lee Cronbach (1951) [Cro51] a proposé l'alpha de Cronbach dans le domaine de la psychologie. Par la suite, Gulliksen (1950) [Gul50], Rasch (1966) [Ras66], Magnusson (1967) [Mag67] et Lord et Novick (1968) [Lor68] ont travaillé sur le modèle du score «vrai ou faux». Ce modèle mesure la capacité d'un individu de répondre à un item, en fonction de son score. En revanche, ce modèle ne prend pas en compte les cas d'items avec plus de deux modalités de réponse. Le modèle polytomique de Rasch est une solution adaptée pour traiter le cas de plus de deux modalités de réponse. Il a été proposé par Masters (1982) [Mas82]. Ce modèle, appelé crédit partiel, est une dérivation du modèle de Rasch.

Il existe de nombreuses études récentes, où les auteurs utilisent le modèle de crédit partiel pour valider des échelles de mesure subjectives, par exemple, the living with chronic illness scale in Parkinson's disease[Amb20], the Burke Lateropulsion Scale[Bir20], the Person-Centred Climate Questionnaire[Wil19]...

Dans la littérature [Tub03, Den17, Cam17, App15], les études de validation psychométrique de l'échelle GOHAI basent leurs analyses sur la cohérence interne (Cronbach alpha), la reliabilité au cours du temps, la validité discriminante et concurrente et la validité du construit. En revanche, Franchignoni fonde ses analyses sur le modèle polytomyque de Rasch, c'est-à-dire sur l'étude de l'unidimensionnalité de l'échelle [Fra10]. Il a pu montrer l'unidimensionnalité de l'échelle GOHAI en passant de 5 modalités à 3 modalités de réponse par regroupement $(0=$ jamais, $1=$ parfois et $2=$ souvent et toujours $)$.

L'unidimensionnalité est l'un des principaux concepts de la théorie de réponse aux items [Van97]. L'unidimensionnalité vérifie qu'on étudie un seul concept (ou une seule dimension). En d'autres 
termes, on étudie l'existence de sous-échelles. D'autre part, l'existence de deux ou plusieurs sousdimensions implique que le concept est divisé en deux ou plusieurs sous-concepts. L'étude de l'unidimensionnalité de l'échelle est donc une étape essentielle dans la validation psychométrique des échelles de qualité de vie [Smi02].

Dans cet article, on étudie l'unidimensionnalité de l'échelle GOHAI. En d'autres termes, on étudie la validité de structure de l'échelle GOHAI selon le modèle de crédit partiel [Mok10].

\section{Patients et méthodes}

\subsection{Population étudiée}

Les données ont été extraites d'une précédente étude transversale, la validation française du GOHAI réalisée en 2000-2001 par Tubert-Jeannin et al. parmi un échantillon de 255 adultes du département du Puy de Dôme (France). Les adultes défavorisés étaient surreprésentés dans cet échantillon et leur âge variait de 18 à 45 ans. L'échantillon a été appelé à suivre des examens médicaux et dentaires par trois caisses d'assurance maladie du département du puy de Dôme. Cette étude a été approuvée par le Comité National Informatique et Liberté de Paris qui a donné son autorisation et fixé les conditions de collecte des informations personnelles pour l'étude.

\subsection{L'échelle GOHAI}

L'échelle GOHAI est composée de 12 items avec 5 modalités de réponse chacun, avec un score de 1 à 5 . Le score le plus élevé correspond à une réponse plus favorable au sens d'une bonne perception de la santé bucco-dentaire - voir échelle en annexe. L'échelle GOHAI est une échelle d'auto évaluation de la santé bucco-dentaire qui a été initialement validée aux États-Unis [Atc90].

\subsection{Méthodologie de la validation psychométrique}

La validation psychométrique d'une échelle de mesure subjective se compose de trois parties: la reliabilité, la sensibilité et la validité de l'échelle.

Il existe deux types de reliabilité. Cohérence interne et reliabilité au cours du temps (ou reproductibilité).

La cohérence interne mesure la cohérence entre les items [Cro51]. Il est mesuré en utilisant l'alpha de Cronbach et les inter-corrélations entre les items et le score total. L'alpha de Cronbach prend une valeur comprise entre 0 et 1 , il est considéré comme acceptable à partir de 0,7[Nun78], cette acceptabilité (>0,7) est controversée par d'autres auteurs [Mes13].

Le coefficient alpha de Cronbach est défini par [Cro51] :

$$
\alpha=\frac{\mathrm{k}}{\mathrm{k}-1}\left(1-\frac{\sum_{i=1}^{k} S_{Y_{i}}^{2}}{S_{X}^{2}}\right)
$$

Où $\mathrm{k}$ est le nombre d'items, $S_{X}^{2}$ est la variance empirique du score total et $S_{Y_{i}}^{2}$ est la variance empirique de l'item $\mathrm{i}$.

La validité de structure confirme la structure postulée ou hypothétique d'un questionnaire. Le lien entre les items appartenant au même domaine ou dimension doit être plus fort que le lien entre les items appartenant à des dimensions différentes. Cette validité est mesurée par le modèle de crédit partiel. 


\subsection{Modèle de crédit partiel (PCM)}

Le modèle de crédit partiel est présenté par Master (1982) [Mas82], le PCM appartient à la famille des modèles polytomiques de Rasch. Ce modèle peut être appliqué à toutes les études avec au moins deux modalités de réponse pour chaque item. Toutes les modalités de réponse sont ordonnées, par exemple, pour un item contenant $\mathrm{m}$ modalité de réponse, 0 est la modalité de réponse la moins favorable et m-1 est la modalité de réponse la plus favorable d'un point de vue psychométrique. Le PCM contient deux types de paramètres, la difficulté de l'item et l'aptitude de l'individue.

Dans le PCM, la probabilité que l'individu j réponde x à l'item i est présentée par :

$$
P_{i j x}=\frac{\exp \sum_{k=0}^{x}\left(\theta_{j}-\delta_{i k}\right)}{\sum_{h=0}^{m_{j}} \exp \sum_{k=0}^{h}\left(\theta_{j}-\delta_{i k}\right)}, \quad x=0,1, \ldots, m_{i}
$$

Avec $P_{i j x}$ est la probabilité que l'individu j réponde x à l'item i, ${ }_{j}$ est l'aptitude de l'individu j, ${ }_{i k}$ est la difficulté de la modalité $\mathrm{k}$ de l'item i.

Les paramètres de difficulté du modèle PCM sont estimés en utilisant le maximum de vraisemblance conditionnel et marginal [Mas97]. Dans le package 'eRm' du logiciel R 3.6.3, le maximum de vraisemblance conditionnelle est utilisé pour obtenir les estimations des paramètres de difficulté du modèle PCM, qui sont ensuite insérés dans les équations de vraisemblance jointe pour estimer les paramètres latents ([Mai07], et ci-dessous, en paragraphe 2.3.1).

\subsubsection{Maximum de vraisemblance conditionnelle}

Les estimations des paramètres du modèle PCM avec la méthode du maximum de vraisemblance jointe sont non-consistants, en raison du grand nombre de paramètres à estimer. Car lorsque le nombre d'individus est important, le nombre de paramètres des aptitudes des individus à estimer augmente, ce qui conduit à une non-consistance [Ney48]. En revanche, la méthode du maximum de vraisemblance conditionnelle résout le problème de la non-consistance car on considère les aptitudes des individus comme des paramètres fixes (les scores $\mathrm{S}$, définis ci-dessous, sont exhaustifs des aptitudes des individus) et seuls les paramètres des items sont estimés [Mas97].

Fixant un individu v, soit $X_{v}=\left(X_{v 1}, \ldots, X_{v K}\right)$

$$
\begin{aligned}
L_{C v}\left(\delta / x_{v}, s_{v}, \theta_{v}\right) & =P\left(X_{v}=x_{v} / \theta_{v}, s_{v}\right) \\
& =\frac{P\left(X_{v}=x_{v} / \theta_{v}\right) \times P\left(S_{v}=s_{v} / \theta_{v}, \delta, X_{v}=x_{v}\right)}{P\left(S_{v}=s_{v} / \theta_{v}\right)}
\end{aligned}
$$

$L_{C v}$ est le maximum de la vraisemblance conditionnelle de $\delta$ sachant $x_{v}, s_{v}, \theta_{v}$ pour l'individu v et $s_{v}$ est le score de l'individu v.

$$
\begin{aligned}
P\left(S_{v}=s_{v} / \theta_{v}\right) & =\sum_{x_{v} \in X^{(s)}} \prod_{i=1}^{K} \frac{\exp \left(x_{v i} \theta_{v}+s_{i x_{v i}}\right)}{\sum_{h=0}^{m_{i}} \exp \left(h \theta_{v}+s_{i h}\right)} \\
& =\sum_{x \in X^{(s v)}} \frac{\exp \left(s_{v} \theta_{v}\right) \times \exp \left(\sum_{i=1}^{K} s_{i x_{v i}}\right)}{\prod_{i=1}^{K}\left[\sum_{h=0}^{m_{i}} \exp \left(h \theta_{v}+\delta_{i h}\right)\right]} \\
& =\frac{\exp \left(s_{v} \theta_{v}\right) \times \gamma_{s}(\delta)}{\prod_{i=1}^{K}\left[K_{i}\left(\delta_{i}, \theta\right)\right]}
\end{aligned}
$$

$\mathrm{X}^{\left(\mathrm{z}_{\mathrm{v}}\right)}={ }_{\mathrm{L}}$ 'ensemble de vecteur possibles, dont la somme des $\mathrm{x}_{\mathrm{v}}$ est égale à $\mathrm{S}_{\mathrm{v}}$, 
autrement dit, tels que $\sum_{i=1}^{K} x_{v i}=s_{v}$ où $x_{v}=\left(x_{v 1}, \ldots, x_{v K}\right)$.

Et, $\gamma_{s_{v}}(\delta)=\sum_{x_{v} \in X^{\left(s_{v}\right)}} \exp \left(\sum_{i=\mathbf{1}}^{K} \delta_{i x_{v i}}\right)$. On en déduit :

$$
\begin{aligned}
& \gamma_{0}(\delta)=1 \\
& \gamma_{1}(\delta)=\exp \left(\delta_{11}\right)+\cdots+\exp \left(\delta_{k 1}\right) \\
& \cdots \\
& \gamma_{k m_{1}}(\delta)=\exp \left(\delta_{1 m_{1}}+\cdots+\delta_{k m_{k}}\right)
\end{aligned}
$$

Et, sachant que $x_{v i}=h$, la somme des autres items est $s_{v}-h$.

$$
\text { Donc, } \gamma_{s_{v}}(\delta)=\sum_{x_{v i}=1}^{m_{i}} \exp \left(\delta_{i x_{n i}}\right) \gamma_{s_{v}-x_{v i}}^{(i)}+\gamma_{s_{v}}^{(i)}
$$

Avec

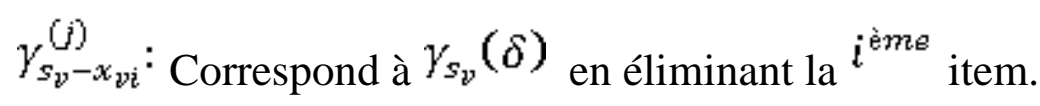

$$
\begin{aligned}
& \gamma_{s_{v}-x_{v i}}^{(j)}=\sum_{x_{v-j} \in X^{\left(s_{v}, x_{v i}\right)}} \exp \left(\sum_{i=1}^{j-1} \delta_{i x_{v i}}+\sum_{i=j+1}^{K} \delta_{i x_{v i}}\right) \\
& K_{i v}\left(\delta_{i}, \theta_{v}\right)=\sum_{h=0}^{m_{i}} \exp \left(h \theta_{v}+\delta_{i h}\right) \\
& P\left(X_{v}=x_{v} / \theta_{v}, S_{v}=s_{v}\right)=\prod_{i=\mathbf{1}}^{K} P\left(X_{v i}=x_{v i} / \theta_{v}, S_{v}=s_{v}\right) \\
& =\prod_{i=\mathbf{1}}^{K} \frac{\exp \left(x_{v i} \theta_{v}+\delta_{i x_{v i}}\right)}{\sum_{h=0}^{m_{i}} \exp \left(h \theta_{v}+\delta_{i h}\right)} \\
& =\frac{\exp \left(s_{v} \theta_{v}\right)+\exp \left(\sum_{i=\mathbf{1}}^{k} \delta_{i x_{v i}}\right)}{\prod_{i=\mathbf{1}}^{k} K_{i v}\left(\delta_{i}, \theta_{v}\right)} \\
& P\left(S_{v}=s_{v} / \theta_{v}, \delta, X_{v}=x_{v}\right)=1 \\
& \text { Alors } L_{C v}\left(\delta / x_{v}, s_{v}, \theta_{v}\right)=\frac{\exp \left(\sum_{i=1}^{K} \delta_{i x_{v i}}\right)}{\gamma_{s_{v}}(\delta)}
\end{aligned}
$$

Pour l'ensemble des observations, le maximum de vraisemblance conditionnelle est :

$$
L_{C}\left(\delta / x_{v}, s_{v}\right)=\prod_{v=1}^{n} \frac{\exp \left(\sum_{i=1}^{K} \delta_{i x_{v i}}\right)}{\gamma_{s_{v}}(\delta)}
$$


Les estimations des paramètres de difficulté de l'item sont finies et uniques, et ces estimations sont consistantes.

Le maximum de vraisemblance jointe est utilisée pour estimer les aptitudes des individus en remplaçant les paramètres de difficulté des items par leurs estimations [Mai07, And95].

$$
s_{v}-\sum_{i=1}^{h} \sum_{h=1}^{m_{i}} \frac{h \exp \left(h \theta_{v}+\hat{\delta}_{i h}\right)}{\sum_{l=0}^{m_{i}} \exp \left(h \theta_{v}+\hat{\delta}_{i l}\right)}=0
$$

\subsubsection{Technique et méthode de validation graphique}

\subsubsection{Graphes caractéristiques des items}

Le graphe caractéristique d'un item présente plusieurs courbes, et chaque courbe correspond à une modalité de réponse de cet item. L'axe d'abscisse est l'aptitude des individus $\left({ }^{\theta}\right)$, et l'axe d'ordonnée est la probabilité de réponse. Ainsi, ces courbes correspondent aux probabilités des modalités de réponse en fonction de l'aptitude des individus. Le point de rencontre de deux courbes qui présente deux modalités consécutives est la difficulté de la modalité $\left(^{\delta}\right)$.

\subsubsection{Courbe d'information}

Le courbe d'information de l'item mesure la quantité d'information fournie par chaque item, après les estimations des paramètres du modèle PCM. Chaque item peut avoir une courbe d'information qui étudie sa variabilité autour de sa valeur centrale par rapport à l'aptitude des individus $\left({ }^{\theta}\right)$. Ces courbes ont une forme gaussienne, la précision de l'item est plus importante lorsque la courbe d'information de cet item est plus serrée autour de sa valeur centrale. Les items faciles donnent plus d'informations pour les individus ayant des aptitudes faibles, et les items difficiles donnent plus d'informations pour les individus ayant des aptitudes importantes. De plus, une courbe d'information de l'échelle mesure la quantité d'informations fournies par l'échelle avec des individus ayant différents niveaux d'aptitude [Rou99].

\subsubsection{Graphe des valeurs propres}

Le graphe des valeurs propres montre les principales dimensions de l'échelle à étudier, il est plutôt utilisé dans les analyses factorielles. Ainsi, le graphe des valeurs propres donne une image préliminaire de l'étude de la validité de structure, une valeur propre beaucoup plus grande que les autres va dans le sens l'existence d'une dimension dominante.

\subsubsection{Qualité de l'ajustement}

Dans le PCM, on test l'ajustement des items et l'ajustement des individus. L'ajustement des items décrit si les items ont le même concept, s'il existe d'autres sous-concepts ou si un item est ambigu. L'ajustement individuel identifie les individus dont les réponses ne suivent pas le schéma général des réponses.

En validité de structure, on s'intéresse à l'ajustement des items pour étudier l'homogénéité de l'échelle. En d'autres termes, pour étudier l'unidimensionnalité. On utilise la statistique d'ajustement au carré moyen pour tester l'ajustement du modèle, où on calcule la moyenne de la différence au carré entre la réponse observée et attendue du PCM. La théorie des modèles de Rasch définit deux types d'ajustement, l'outfit et l'infit [Bon07].

$$
\text { Outfit }_{i}=\frac{1}{n} \sum_{v=1}^{n} \frac{\left(x_{v i}-E_{v i}\right)^{2}}{\operatorname{Var}\left(x_{v i}\right)}
$$




$$
\begin{aligned}
& \text { Infit } t_{i}=\frac{\sum_{v=1}^{n}\left(x_{v i}-E_{v i}\right)^{2}}{\sum_{v=1}^{n} \operatorname{Var}\left(x_{v i}\right)} \\
& \operatorname{Var}\left(x_{v i}\right)=\sum_{k=0}^{m_{i}}\left(k-E_{v i}\right)^{2} \times P_{i j k}
\end{aligned}
$$

Où Outfit $_{i}$ est l'outfit de l'item i, Infit $t_{i}$ est l'infit de l'item i, ${ }_{v i}$ est la réponse de l'individu v à l'item $\mathrm{i}, E_{v i}$ est la réponse attendue de l'individu v à l'item i et $\operatorname{Var}\left(x_{v i}\right)$ est la variance empirique de $X_{v i}$.

Ces deux statistiques ont une valeur attendue égale à un sous le PCM. Une valeur d'infit ou d'oufit pour un item proche de zéro, ou bien supérieure à un, indique que l'item a un mauvais ajustement avec le modèle. Plus précisément, une valeur d'outfit ou une valeur d'infit pour un item inférieure à 0,5 ou supérieure à 1,5 indique que l'item a un mauvais ajustement avec le modèle [Lin02].

\section{Résultats}

L'échantillon total comprend 260 individus. Soixante-cinq pour cent des individus sont âgées de 30 ans ou plus. Près de la moitié des individus (49\%) sont des femmes. Vingt-neuf pour cent des individus ont déclaré avoir terminé leurs études secondaires. Près de $60 \%$ des individus vivent en zone urbaine (population> 5000 habitants). Quarante et un pour cent des individus sont mariés ou vivent en couple tandis que les autres sont célibataires (avec ou sans enfants). Près de la moitié (47\%) des individus sont sans emploi [Tub03].

On utilise le package eRm du logiciel R 3.6.3 dans l'analyse statistique.

\subsection{Reliabilité de l'échelle}

Le coefficient alpha de Cronbach est de 0,86. Il montre un degré significatif de cohérence interne entre les items.

\subsection{Analyse du modèle de crédit partiel}

On a exclu 30 individus de l'étude du modèle de crédit partiel en raison du grand nombre de réponses manquantes parmi ces individus (absence de données Questionnaire QDV).

Pour réaliser le modèle de crédit partiel, on a besoin d'au moins 60 individus (nombre d'items multiplié par le nombre de modalités de réponse) [Mas82]. Ainsi, le nombre d'individus est suffisant pour appliquer le modèle de crédit partiel $(\mathrm{n}=230)$.

Le threshold correspond à la difficulté de la modalité de réponse, l'item le plus simple est l'item 6, tandis que l'item le plus difficile est l'item 12. En d'autres termes, la probabilité qu'un individu donne une réponse favorable à l'item 6 est la plus grande, et la probabilité qu'un individu donne une réponse défavorable à l'item 12 est la plus importante (tableau 1). 


\begin{tabular}{|c|l|l|l|l|l|}
\hline Items & \multicolumn{1}{|c|}{ Location } & \multicolumn{1}{|c|}{ Threshold 1 } & \multicolumn{1}{|c|}{ Threshold 2 } & \multicolumn{1}{|c|}{ Threshold 3 } & \multicolumn{1}{c|}{ Threshold 4 } \\
\hline Item 1 & -0.27585 & -0.62399 & -0.48300 & 1.64293 & -1.63932 \\
\hline Item 2 & 0.11364 & -0.00549 & -0.34573 & 1.28587 & -0.48009 \\
\hline Item 3 & -0.10627 & 1.41088 & -0.84129 & -0.03607 & -0.95861 \\
\hline Item 4 & -0.40062 & 0.31947 & -1.21033 & 1.06930 & -1.78090 \\
\hline Item 5 & 0.33711 & 0.47915 & -0.21010 & 1.15417 & -0.07479 \\
\hline Item 6 & -0.82189 & -1.86439 & -0.47886 & 0.47415 & -1.41847 \\
\hline Item 7 & 0.66552 & 0.15409 & 0.95347 & 0.48064 & 1.07390 \\
\hline Item 8 & -0.13453 & -1.90622 & -0.01581 & 1.25922 & 0.12468 \\
\hline Item 9 & 0.57414 & -0.37082 & 0.29095 & 1.26326 & 1.11319 \\
\hline Item 10 & 0.18803 & -0.58735 & 0.41817 & 1.05855 & -0.13723 \\
\hline Item 11 & -0.23744 & -0.30538 & -0.25431 & 0.61345 & -1.00349 \\
\hline Item 12 & 0.82282 & -0.45829 & 0.13660 & 2.19124 & 1.42171 \\
\hline
\end{tabular}

Tableau 1. Difficultés des items

Le graphe des valeurs propres montre une valeur propre plus importante que les autres, ce qui vérifie l'existance d'une dimension dominante (Figure 1). Ainsi, ce graphe renforce l'idée de unidimenssionalité de l'échelle.

Les courbes d'informations des items montrent que tout les items ont une bonne précision, on ne trouve aucune courbe plate. Les items 1, 4, et 6 donnent plus d'information sur les individus ayant une aptitude faible, tandis que les items 7, 9 et 12 donnent plus d'information sur les individus ayant une aptitude importante (figure 2). La courbe d'information de l'échelle montre une bonne précision globale, et porte le maximum d'information pour les individus avec des niveaux moyens d'aptitude (figure 2).

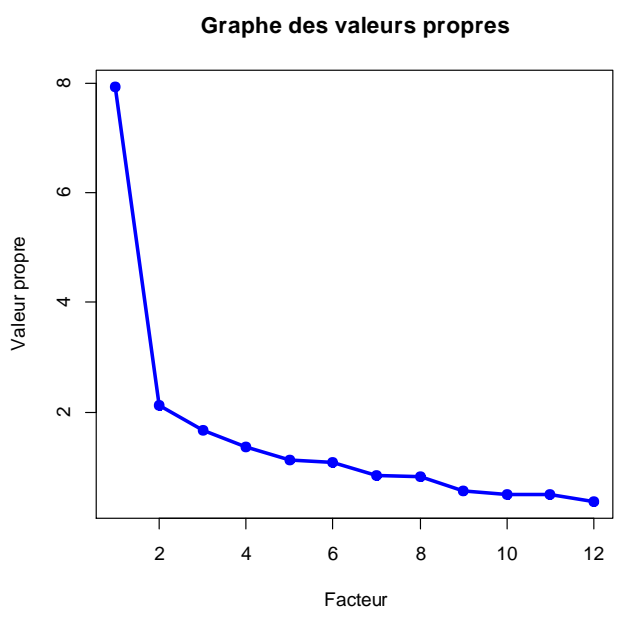

Figure 1. Graphe des valeurs propres 

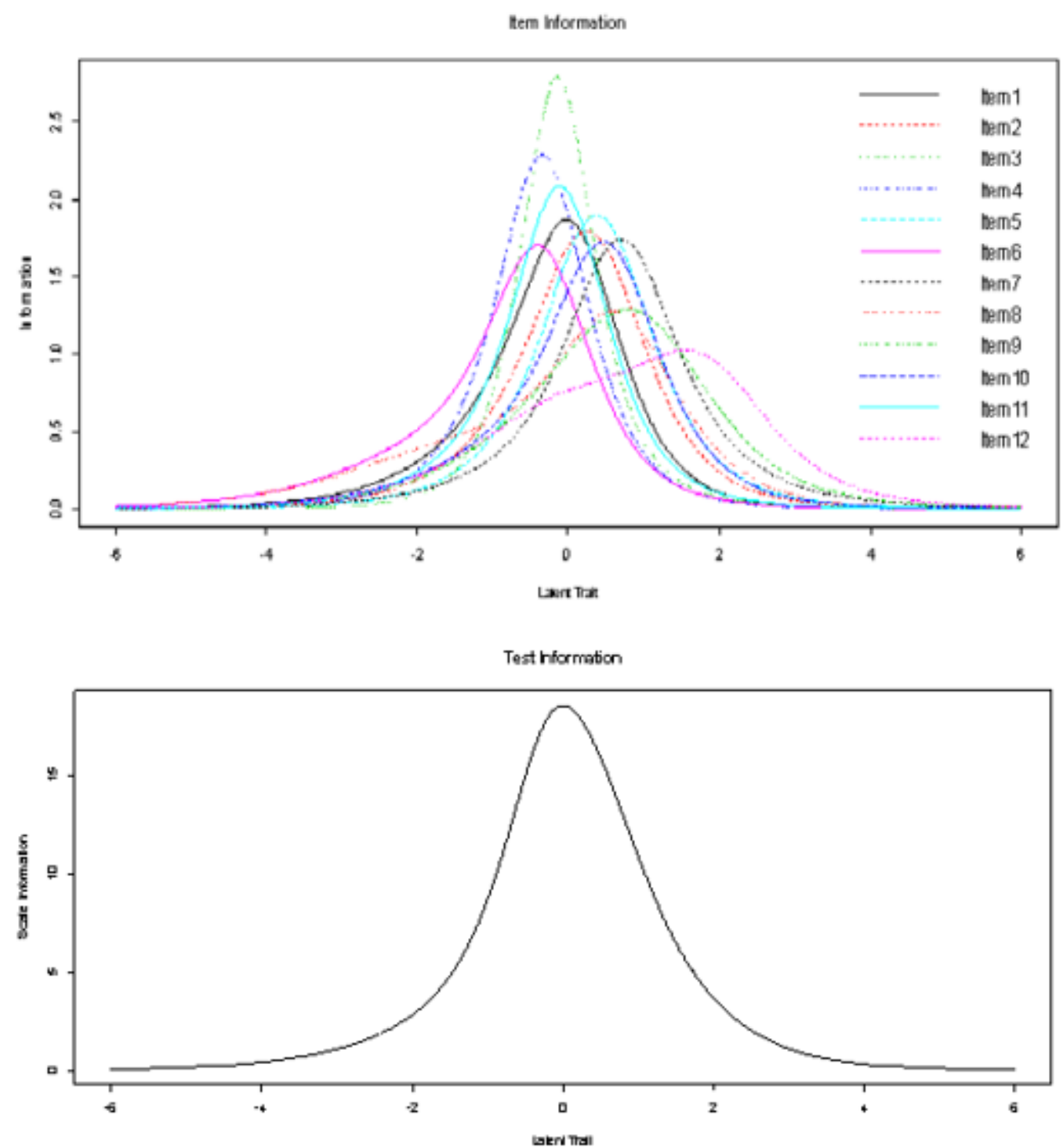

Figure 2. Courbes d'informations

On remarque à partir des graphes caractéristiques des items (figure 3), qu'on a au moins une courbe pour chaque item toujours en dessous des autres courbes. Ce résultat garantit la présence de modalités de réponse rarement sélectionnés pour tous les items. 
ICC plot for item $\times 1$

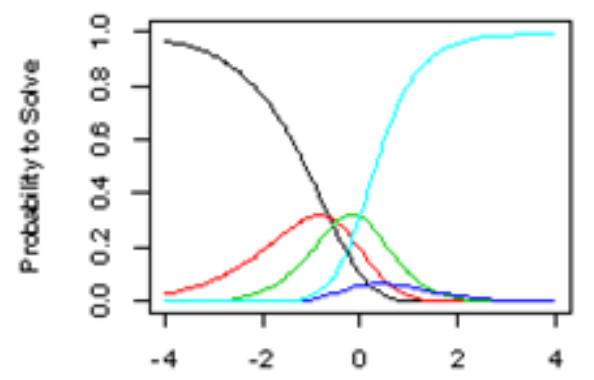

Latent Dimension

IC C plot for item X4
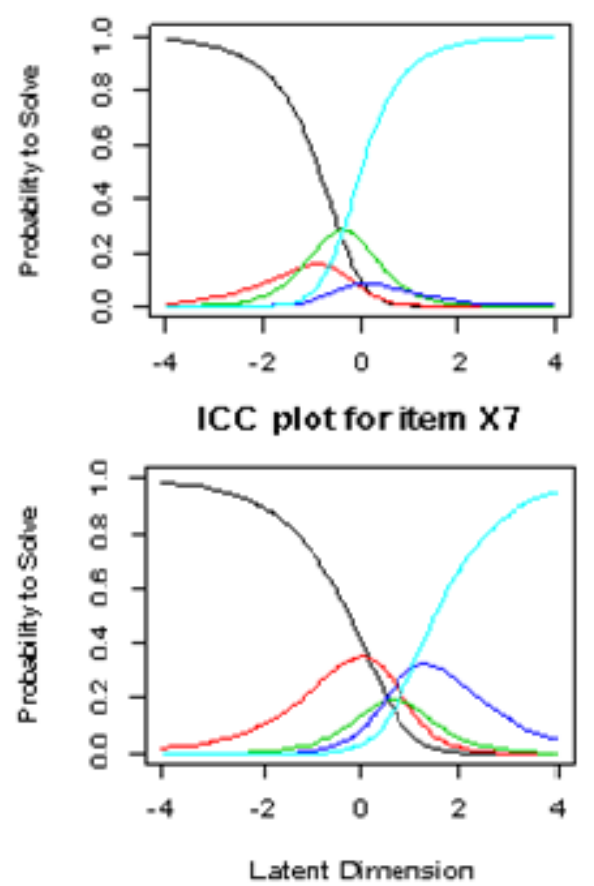

IC C plot for item $\times 10$

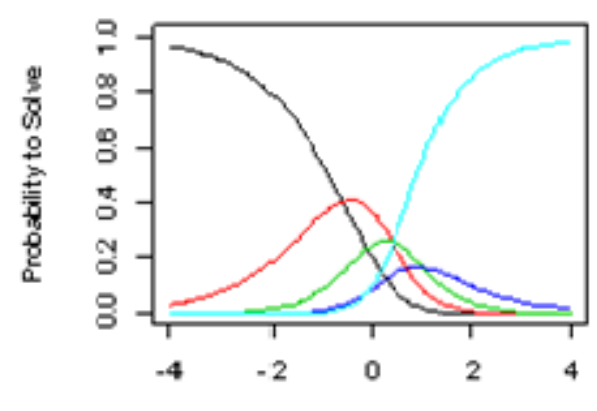

Laternt Dimension
ICC plot for item X2

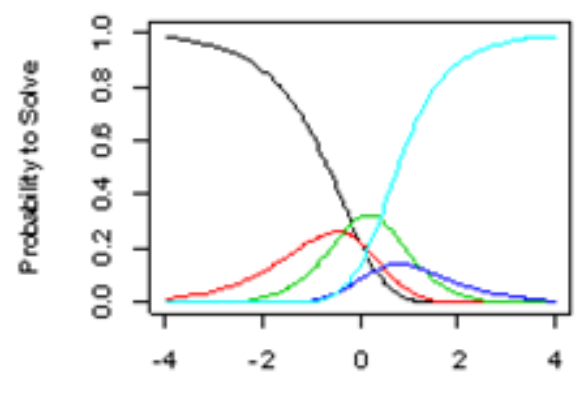

Latert Dimension

ICC plot for item $\times 5$
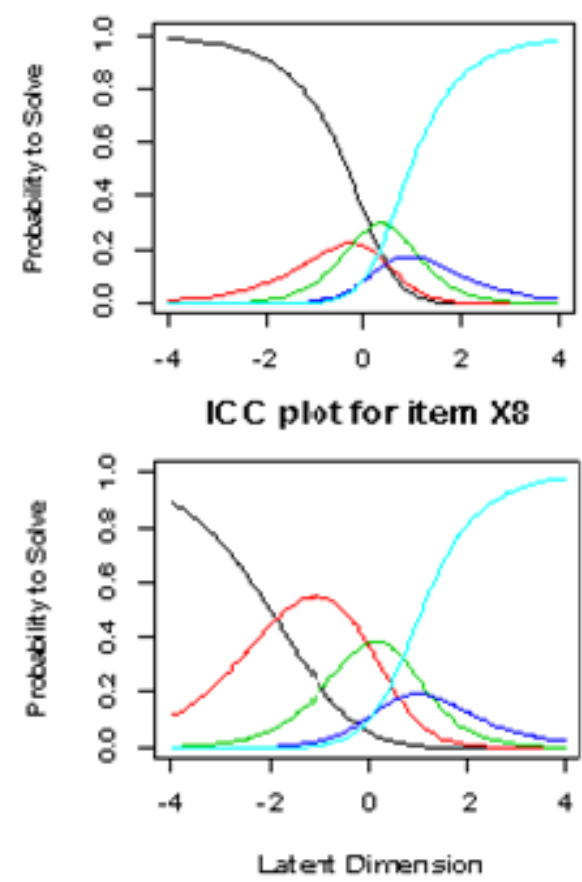

ICC plot for item $\times 11$

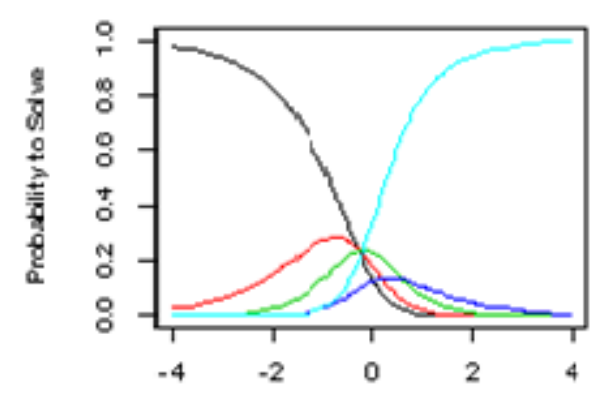

Latent Dimension

\section{ICC plot for item X3}

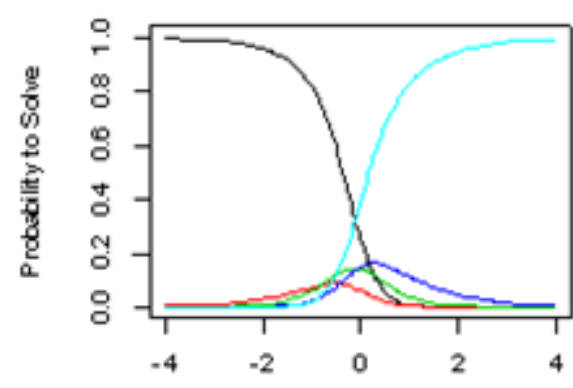

Latent Dimension

IC C plot for item X6
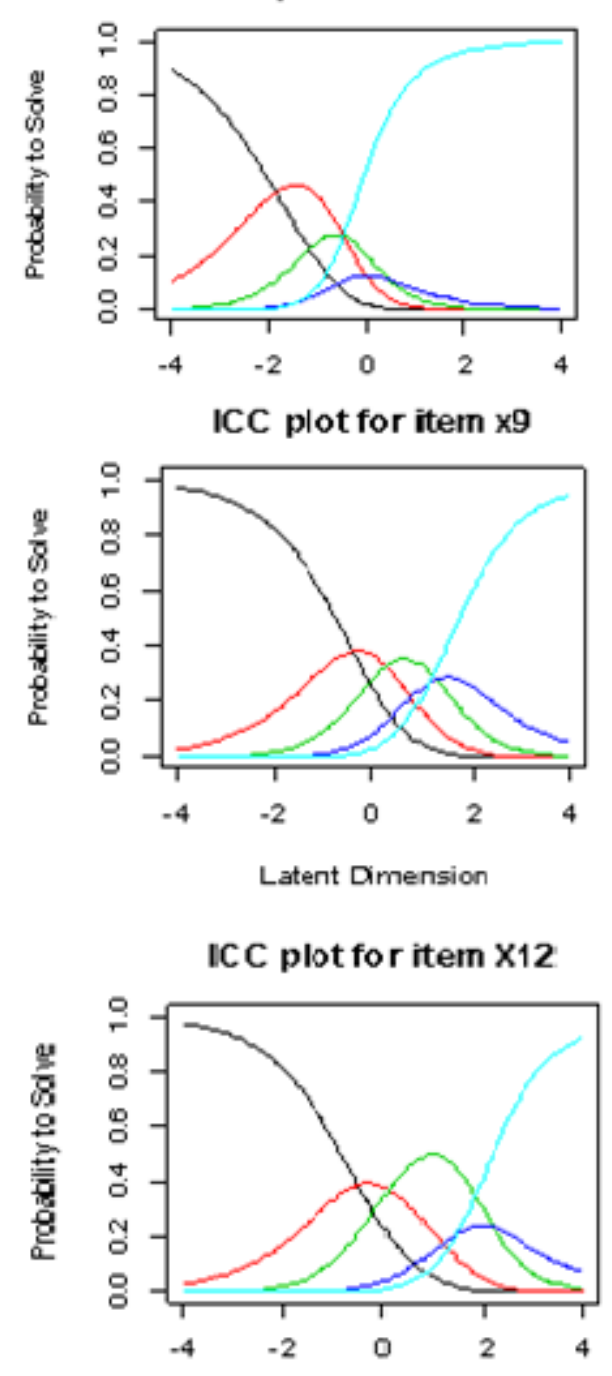

Laternt Dimension

Figure 3. Graphes caractéristiques des items

Tous les items de l'échelle GOHAI montrent un bon ajustement (MSQ outfit et MSQ infit entre 0.5 et 1.5) avec le modèle de crédit partiel, sauf que l'item 3 a un mauvais ajustement avec le modèle (MSQ supérieur à 1,5), ce qui indique qu'il est difficile d'attendre les réponses des individus à l'item 3 (tableau 2). 


\begin{tabular}{|c|l|l|}
\hline Items & \multicolumn{1}{|c|}{ Outfit MSQ } & \multicolumn{1}{c|}{ Infit MSQ } \\
\hline Item 1 & 0.514 & 0.710 \\
\hline Item 2 & 0.847 & 0.757 \\
\hline Item 3 & $\mathbf{1 . 8 1 4}$ & $\mathbf{1 . 5 0 1}$ \\
\hline Item 4 & 0.547 & 0.743 \\
\hline Item 5 & 1.422 & 1.304 \\
\hline Item 6 & 0.624 & 0.791 \\
\hline Item 7 & 0.974 & 0.995 \\
\hline Item 8 & 1.092 & 1.099 \\
\hline Item 9 & 0.944 & 0.913 \\
\hline Item 10 & 0.584 & 0.678 \\
\hline Item 11 & 0.509 & 0.650 \\
\hline Item 12 & 1.089 & 1.105 \\
\hline
\end{tabular}

Tableau 2. Statistiques d'ajustement des items

De ce résultat, on peut conclure que les items de l'échelle GOHAI ont le même concept, sauf l'item 3 qui ne peut pas être considéré comme une sous-dimension.

\section{Discussion}

\subsection{Unidimensionnalité de l'échelle GOHAI}

Dans cet article, on teste l'unidimensionnalité de l'échelle GOHAI à l'aide des données de TubertJannin. On a montré que les items de l'échelle GOHAI ont un bon ajustement, à l'exception de l'item 3, au modèle de crédit partiel. Ainsi on peut conclure que les items de l'échelle GOHAI sont homogènes et ont le même concept, en d'autres termes, on peut conclure l'unidimensionnalité de l'échelle GOHAI.

\subsection{Analyse factorielle}

L'analyse factorielle est utilisée pour étudier la validité de structure, l'idée principale de cette méthode est de réduire autant que possible le nombre de dimensions, où au début chaque variable (item) correspond à une dimension. Ainsi, cette méthode aboutit en paratiques à des échelles multidimensionnelles dans les études de validation psychométrique, ce qui est remarquable dans la littérature, par exemple, "the Second Victim Experience and Support Tool" (C-SVEST)[Che19], "questionnaire to measure post-intensive care syndrome" [Jeo19], "Chinese empathy motivation scale[Zhu19], The Stigma of Suicide Scale" [Lud20]...

\subsection{Le regroupement des modalités de réponse des items}

L'unidimensionnalité est une hypothèse fondamentale dans un modèle de Rasch polytomique. L'échelle GOHAI contient cinq modalités de réponse pour chaque item. Franchignoni [Fra10] et Hardoin [Den17] réduisent le nombre de modalités de réponse à 3. Cette méthode permet d'obtenir une échelle unidimensionnelle. Cependant, on est perplexes quant au choix de cette méthode. En effet, la réduction du nombre de modalités a pour effet de lisser les données, ce qui fait perdre de la sensibilité et de la précision à la mesure subjective. Ainsi, ces auteurs font le choix de perdre des informations et de la finesse d'analyse afin d'avoir une échelle unidimensionnelle. 
D'une part, ces auteurs obtiennent une échelle unidimensionnelle. Mais d'un autre part, en réduisant le nombre de modalités de 5 à 3 , on perd des informations qui cachent une ou plusieurs sousdimensions, ce qui élimine des résultats importants de l'étude.

\subsection{Sensibilité des échelles}

L'étude de la sensibilité au changement fait partie de la validation psychométrique des échelles. En revanche, elle est rarement mesurée dans la plupart des études de validation des échelles de qualité de vie. Cela est dû au manque du temps et au risque de perte de suivi ou de refus de participation des individus.

\subsection{Protocole de validation psychométrique}

La validation psychométrique des échelles subjectives est un processus composé de trois étapes : reliabilité, validité et sensibilité. En revanche, on ne trouve pas, dans la littérature, une méthode commune de la validation des échelles de mesure subjective. La littérature est remplie d'études qui calculent simplement les alphas de Cronbach pour valider les échelles. Il est important de noter que l'utilisation, seule, de cette statistique surestime les capacités de leurs échelles. En effet, ces études sous-exploitent leurs résultats et manquent de finesse et a fortiori de précision.

\section{Conclusion}

Le modèle de crédit partiel montre que onze des douze items de l'échelle GOHAI ont un bon ajustement, pour cela l'échelle GOHAI est considérée comme unidimensionnelle. La validité de structure n'a pas été étudiée par les auteurs lors de la validation initiale de cette échelle. Dans cet article, on a complété cette pièce manquante de la validation psychométrique de la version française de l'échelle GOHAI.

Dans la littérature, si la validation du contenu semble parfois perdue, la validité de structure est souvent omise. Cependant, on pense que la validation psychométrique des échelles mérite toute l'attention des chercheurs et doit être complète. Ces étapes de validation psychométrique montrent que l'on prend en compte tous les attributs caractéristiques que l'on souhaite évaluer, et examine le niveau de compréhension du questionnaire par les patients, et permet une meilleure compréhension du sens réel du concept mesuré par l'échelle en distinguant cela à partir de concepts connexes et que notre échelle explique clairement le concept envisagé.

Si on a montré que la validité de la structure est importante, il n'en reste pas moins que la validation du contenu des échelles est essentielle et constitue la base de toute analyse et interprétation des observations. La validation psychométrique est comme un puzzle, chaque étape de la validation psychométrique rend l'image plus claire, dont le but ultime est de produire suffisamment d'informations pour aider à améliorer la santé subjective des patients. 


\subsection{Annexe 1 : Items de l'échelle GOHAl, en français et en anglais.}

\section{Ces trois dernier mois}

\section{In the past three months}

1-Avez-vous limité la quantité, ou le genre des aliments que vous mangez en raison de problèmes avec vos dents ou vos appareils dentaires?

How often did you limit the kinds or amounts of food you eat because of problems with your teeth or denture?

2-Avez-vous eu des difficultés pour mordre ou mastiquer certains aliments durs tels que de la viande ou une pomme? How often did you have trouble biting or chewing any kinds of food, such as a firm meat or apples?

3-Avez-vous pu avaler confortablement?

How often were you able to swallow comfortably?

4-Vos dents ou vos appareils dentaires vous ont-ils empêché(e) de parler comme vous vouliez?

How often have your teeth or dentures prevented you from speaking the way you wanted?

5-Avez-vous pu manger de tout (sans ressentir une sensation d'inconfort)?

How often were you able to eat anything without feeling discomfort?

6-Avez-vous limité vos contacts avec les gens à cause de l'état de vos dents ou de vos appareils dentaires?

How often did you limit contacts with people because of the condition of your teeth or dentures?

7-Avez-vous été satisfait(e) ou content(e) de l'aspect de vos dents, de vos gencives ou de vos appareils dentaires?

How often were you pleased or happy with the appearance of your teeth, gums or dentures?

8-Avez-vous pris un (des) médicament(s) pour soulager la douleur ou une sensation d'inconfort dans votre bouche?

How often did you use medication to relieve pain or discomfort around your mouth?

9-Vos problème de dent, de gencive ou d'appareil dentaire) vous ont-ils inquiété(e) ou préoccupé(e) ?

How often were you worried or concerned about the problems with your teeth, gums or dentures?

10-Vous-êtes senti(e) gêné(e) ou mal à l'aise, à cause de problèmes avec vos dents, vos gencives ou vos appareils dentaires) ?

How often did you feel nervous or self-conscious because of problems with your teeth, gums or dentures?

11-Avez-vous éprouvé de l'embarras pour manger devant les autres à cause de vos dents (ou vos appareils dentaires) ? How often did you feel uncomfortable eating in front of people because of problems with your teeth or dentures?

12-Vos dents ou vos gencives ont-elles été sensibles au froid, au chaud, ou aux aliments sucrés? How often were your teeth or gums sensitive to hot, cold or sweet foods? 


\subsection{Annexe 2 : Code du modèle de crédit partiel avec le logiciel R 3.6.3 :}

\#\#modèle de Crédit Partial (PCM)

library $(\mathrm{eRm})$

raschdata $=$ Data

res.pcm $=$ PCM(raschdata $)$

summary(res.pcm)

pres.pcm $=$ person . parameter $($ res.pcm $)$

\#\#aptittudes des individus

Thetta $=$ person $\cdot$ parameter $($ res.pcm $) \$$ theta.table[,-3]

Thetta

itemfit(pres.pcm)\#\# statistiques d'ajustement des items thresholds(res.pcm)\#\# les estimations de la difficulté des items plotPImap(res.pcm, sorted = TRUE)\#\#plan des difficultés des items plotICC(res.pcm, item. subset=1, mplot = FALSE, legpos = FALSE, ask = FALSE)\#\# graphique pour chaque item plotINFO(res.pcm, type $=$ "item", theta $=$ seq(-6, 6, length.out = 1001L),legpos = "below")\#\#Courbe d'information

\section{Bibliography}

[Nun78] Nunnally, J.C.: An Overview of Psychological Measurement. In: Wolman, B.B. (ed.) Clinical Diagnosis of Mental Disorders: A Handbook. Springer US, Boston, MA, 1978.

[Pop00] Pope, C., Ziebland, S., Mays, N.: Analysing qualitative data. BMJ. 320, 114-116, 2000.

[Bla19] Blanc, G.: Validation des propriétés psychométriques d'une échelle d'évaluation des qualités relationnelles en consultation maïeutique. HAL, 2019.

[Tub03] Tubert-Jeannin, S., Riordan, P.J., Morel-Papernot, A., Porcheray, S., Saby-Collet, S.: Validation of an oral health quality of life index (GOHAI) in France. Community Dent. Oral Epidemiol. 31, 275-284, 2003.

[Bel20] Bellier, A., Chaffanjon, P., Krupat, E., Francois, P., Labarère, J.: Cross-cultural adaptation of the 4-Habits Coding Scheme into French to assess physician communication skills. PloS One. 15, e0230672, 2020.

[Che19] Chen, J., Yang, Q., Zhao, Q., Zheng, S., Xiao, M.: Psychometric validation of the Chinese version of the Second Victim Experience and Support Tool (C-SVEST). J. Nurs. Manag. 27, 1416-1422, 2019.

[Tan04] Tan, G., Jensen, M.P., Thornby, J.I., Shanti, B.F.: Validation of the Brief Pain Inventory for chronic nonmalignant pain. J. Pain. 5, 133-137, 2004.

[Moy17] Moya-Suárez, A.B., Morales-Asencio, J.M., Aranda-Gallardo, M., Enríquez de Luna-Rodríguez, M., CancaSánchez, J.C.: Development and psychometric validation of a questionnaire to evaluate nurses' adherence to recommendations for preventing pressure ulcers (QARPPU). J. Tissue Viability. 26, 260-270, 2017.

[Atc90] Atchison, K.A., Dolan, T.A.: Development of the Geriatric Oral Health Assessment Index. J. Dent. Educ. 54, 680-687, 1990.

[Den17] Denis, F., Hamad, M., Trojak, B., Tubert-Jeannin, S., Rat, C., Pelletier, J.-F., Rude, N.: Psychometric characteristics of the "General Oral Health Assessment Index (GOHAI) " in a French representative sample of patients with schizophrenia. BMC Oral Health. 17, 2017.

[Spe07] Spearman, C.: Demonstration of Formula for True Measurement of Correlation. Am. J. Psychol. 18, 161-169, 1907.

[Cro51] Cronbach, L.J.: Coefficient alpha and the internal structure of tests. Psychometrika. 16, 297-334, 1951.

[Gul50] Gulliksen, H.: Intrinsic validity. Am. Psychol. 5, 511-517, 1950.

[Ras66] Rasch, G.: An Item Analysis Which Takes Individual Differences into Account. Br. J. Math. Stat. Psychol. 19, 4957,1966 .

[Mag68] Magnusson, David. Test theory. Reading, Mass.: Addison-Wesley Publishing Company, 1967.

[Lor68] Lord, M.R.N., Allan Birnbaum Frederick M.: Statistical Theories of Mental Test Scores. Addison-Wesley Publishing Company, Reading, Mass, 1968.

[Mas82] Masters, G.N.: A rasch model for partial credit scoring. Psychometrika. 47, 149-174, 1982. 
[Amb20] Ambrosio, L., Rodriguez-Blazquez, C., Ayala, A., Forjaz, M.J.: Rasch analysis of the living with chronic illness scale in Parkinson's disease. BMC Neurol. 20, 2020.

[Bir20] Birnbaum, M., Brock, K., Parkinson, S., Burton, E., Clark, R., Hill, K.D.: Rasch analysis of the Burke Lateropulsion Scale (BLS). Top. Stroke Rehabil. 0, 1-8, 2020.

[Wil19] Wilberforce, M., Sköldunger, A., Edvardsson, D.: A Rasch analysis of the Person-Centred Climate Questionnaire - staff version. BMC Health Serv. Res. 19, 2019.

[Cam17] Campos, J.A.D.B., Zucoloto, M.L., Bonafé, F.S.S., Maroco, J.: General Oral Health Assessment Index: A new evaluation proposal. Gerodontology., 2017.

[App15] Appukuttan, D.P., Vinayagavel, M., Balasundaram, A., Damodaran, L.K., Shivaraman, P., Gunasshegaran, K.: Linguistic Adaptation and Psychometric Properties of Tamil Version of General Oral Health Assessment Index-Tml. Ann. Med. Health Sci. Res. 5, 413-422, 2015.

[Fra10] Franchignoni, M., Giordano, A., Levrini, L., Ferriero, G., Franchignoni, F.: Rasch analysis of the Geriatric Oral Health Assessment Index. Eur. J. Oral Sci. 118, 278-283, 2010.

[Van97] Van Der Linden W.J., Hambleton R.K.: Handbook of Modern Item Response Theory. Springer-Verlag, New York, 1997.

[Smi02] Smith, E.V.: Detecting and evaluating the impact of multidimensionality using item fit statistics and principal component analysis of residuals. J. Appl. Meas. 3, 205-231, 2002.

[Mok10] Mokkink, L.B., Terwee, C.B., Patrick, D.L., Alonso, J., Stratford, P.W., Knol, D.L., Bouter, L.M., de Vet, H.C.W.: The COSMIN study reached international consensus on taxonomy, terminology, and definitions of measurement properties for health-related patient-reported outcomes. Journal of Clinical Epidemiology. 63, 737-745, 2010.

[Mes13] Mesbah, M. (2013). From Measurement to Analysis. In "Rasch Models in Health" Editors: Christensen, K.B., Kreiner, S., Mesbah, M, Wiley, London. Chapter 13.

[Mas97] Masters, G.N., Wright, B.D.: The Partial Credit Model. In: van der Linden, W.J. and Hambleton, R.K. (eds.) Handbook of Modern Item Response Theory. pp. 101-121. Springer, New York, NY, 1997.

[Mai07] Mair, P., and Hatzinger, R.: Extended Rasch modeling: The eRm package for the application of IRT models in R. Journal of Statistical Software, 20(9), 1-20, 2007.

[Rou99] Rouxel, G.: Modèles de Réponse à l'Item pour items polytomiques: exemple d'utilisation du logiciel MULTILOG. Psychologie et Psychométrie, 113-130, 1999.

[And95] Andersen, E.B.: Polytomous Rasch Models and their Estimation. In: Fischer, G.H. and Molenaar, I.W. (eds.) Rasch Models: Foundations, Recent Developments, and Applications. pp. 271-291. Springer, New York, NY, 1995.

[Ney48] Neyman, J., Scott, E.L.: Consistent Estimates Based on Partially Consistent Observations. Econometrica. 16, 1$32,1948$.

[Bon07] Bond, T.G., Fox, C.M.: Applying the Rasch model: Fundamental measurement in the human sciences, 2nd ed. Lawrence Erlbaum Associates Publishers, Mahwah, NJ, US, 2007.

[Lin02] Linacre, J.: A User"s Guide to Winsteps: Rasch-Model Computer Program. Chicago: MESA Press, 2002.

[Jeo19] Jeong, Y.J., Kang, J.: Development and validation of a questionnaire to measure post-intensive care syndrome. Intensive Crit. Care Nurs. 55, 102756, 2019.

[Zhu19] Zhu, Y., Zhan, Y.-C., Zhu, J.-M., Huang, L., Zhang, L., Zhang, M., Li, B.-K.: The development and psychometric validation of a Chinese empathy motivation scale. J. Clin. Nurs. 28, 2599-2612, 2019.

[Lud20] Ludwig, J., Liebherz, S., Dreier, M., Härter, M., von dem Knesebeck, O.: [The Stigma of Suicide Scale: Psychometric Validation of the German Short Version (SOSS-SF-D)]. Psychiatr. Prax. 47, 433-439, 2020.

[Den17] Denis, F., Bizien, P., Tubert-Jeannin, S., Hamad, M., Trojak, B., Rude, N., Hardouin, J.-B.: A Rasch Analysis between Schizophrenic Patients and the General Population. Transl. Neurosci. 8, 139-146, 2017. 